\title{
Traduire
}

Une autre perspective sur r tr traduction

Revue française de la traduction

$240 \mid 2019$

Quand la politique s'en mêle

\section{Jeanne et Kurt Stern, des traducteurs en pleine guerre froide}

Perrine Val

\section{(2) OpenEdition \\ Journals}

Édition électronique

URL : http://journals.openedition.org/traduire/1722

DOI : 10.4000/traduire.1722

ISSN : 2272-9992

Éditeur

Société française des traducteurs

Édition imprimée

Date de publication : 20 juin 2019

Pagination : 84-95

ISSN : 0395-773X

\section{Référence électronique}

Perrine Val, « Jeanne et Kurt Stern, des traducteurs en pleine guerre froide », Traduire [En ligne], 240 |

2019, mis en ligne le 29 juin 2019, consulté le 05 février 2020. URL : http://journals.openedition.org/ traduire/1722 ; DOI : 10.4000/traduire.1722 


\section{Jeanne et Kurt Stern, des traducteurs en pleine guerre froide}

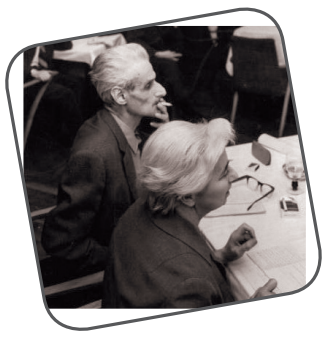

Perrine Val

En dépit du rideau de fer, dont on célébrera à l'automne 2019 le trentième anniversaire de la disparition, la frontière entre l'Est et l'Ouest de l'Europe se révélait parfois plus poreuse que ce que laissaient présupposer les tensions géopolitiques de la guerre froide au cours de la seconde moitié $d u x x^{e}$ siècle. Pour effectuer des échanges - matériels comme intellectuels -, les chemins à emprunter s'avéraient simplement plus tortueux. Les traducteurs jouèrent à cet égard un rôle essentiel, puisqu'ils favorisèrent la diffusion d'œuvres potentiellement inaccessibles aux lecteurs de l'autre côté du mur de Berlin. Les enjeux de la traduction dans ce contexte alternant tensions sous-jacentes, affrontements directs, apaisement et tentatives de rapprochement sont dès lors particulièrement complexes. Dans l'ouvrage Traduire sous contraintes. Littérature et communisme (1947-1989), loana Popa rappelle que les traductions d'œuvres littéraires issues des pays sous tutelle soviétique pouvaient autant relever de la propagande du régime officiel que soutenir des actes de subversion, en permettant par exemple à un auteur censuré de rencontrer un lectorat étranger réceptif:

La traduction participe ainsi des moyens de subversion permettant de remettre en cause ces régimes, alors que toute forme de contes- 
tation légale est supprimée. [...] Non sans dilemmes, incertitudes ou risques, la possibilité d'accéder à un espace intellectuel étranger (ici, occidental) par la traduction de son ceuvre sera progressivement envisagée, en raison de la notoriété qu'elle peut y procurer, comme un moyen de protection des écrivains face aux normes de persécution qu'ils encourent dans leur pays'.

Il en va de même pour les ouvrages venus de l'Ouest: étroitement soumis au verdict des comités de censure, ils rencontrent parfois l'approbation de la politique socialiste lorsqu'ils critiquent par exemple le système capitaliste. À l'inverse, certaines œuvres occidentales désapprouvées par les partis au pouvoir continuent à circuler clandestinement à l'Est ${ }^{2}$. De la même façon que la frontière entre l'Est et l'Ouest était poreuse, la ligne entre les ouvrages interdits, tolérés ou approuvés pouvait, elle aussi, être fluctuante.

À cet égard, la situation de la République démocratique allemande (RDA) s'illustre par sa singularité. Le Mur sépare en effet deux entités qui partagent non seulement la même histoire, mais aussi la même langue. Pour décrire cet espace commun, Matthias Steinle utilise le concept de "dispositif de la division allemande ${ }^{3}$ » afin d'appréhender la situation paradoxale d'une Allemagne certes divisée, mais pourtant unie par son passé, sa langue et aussi via les médias. La RDA construit très largement son identité en opposition à sa voisine ovest-allemande. Cette affirmation d'un nationalisme est-allemand bien distinct passe dans un premier temps par la mise sous tutelle soviétique puis par l'instauration d'un régime socialiste, mais également par des processus complémentaires, tels qu'une réécriture de l'Histoire ou une attention particulière portée à la langue et au vocabulaire ${ }^{4}$. Qu'il s'agisse d'œuvres littéraires ou audiovisuelles, il est frappant de constater l'importance attachée à leur traduction en allemand. Se penchant sur la réception d'Émile Zola en RDA, Aurélie Barjonet emploie ainsi les termes "révision» et «réhabilitation» pour souligner à quel point les traductions est-allemandes des Rougon-Macquart ne se contentent pas de rendre accessible l'œuvre du romancier naturaliste au lectorat

\footnotetext{
1. Popa, p. 8.

2. La censure de romans français dans les régimes communistes constituait l'un des objets du colloque Inventer la littérature française dans un État totalitaire, Paris, Maison de la recherche, 25-26 octobre 2018.

3. "Dispositiv der deutschen Teilung», Steinle, p. 25.

4. Jarausch, p. 19-34.
} 
est-allemand, mais ambitionnent plus largement de valoriser l'image de Zola et de l'intégrer au patrimoine socialiste dont se réclame la RDA. De même, Sylvie Lindeperg revient sur la version est-allemande du film Nuit et Brouillard (Alain Resnais 1956), qui diffère volontairement de celle diffusée en République fédérale d'Allemagne (RFA), quitte à comporter "plusieurs omissions, modifications et contresens graves ${ }^{5} \gg$, en particulier dans l'épilogue du film ${ }^{6}$. Ces deux cas démontrent que ce qui se joue ici relève davantage de l'adaptation que de la traduction: pour les romans de Zola comme pour le film de Resnais, la RDA cherche à intégrer ces œuvres dans son propre système de référence, même si cela implique de trahir leur sens original. La tâche des traducteurs est-allemands est donc particulièrement ardue, puisque leur travail consiste non seulement à s'insérer dans le système complexe d'échanges entre l'Ouest et l'Est, mais aussi à réaffirmer en permanence la singularité de l'identité est-allemande.

Rappelons par ailleurs la situation particulière de la RDA à l'égard de l'étranger. Tout au long de son existence, cette «autre» Allemagne ne cessa d'osciller entre la volonté de gagner en reconnaissance sur la scène internationale et l'adoption d'une position défensive face aux pays occidentaux. Que ce soit sur le plan politique ou culturel, la RDA est toujours mue par le besoin de proclamer la légitimité de son existence pour accroître sa visibilité à l'extérieur de ses frontières. Elle recourt parfois à des moyens conséquents pour atteindre ce but. La diffusion d'informations à son propre sujet dans de multiples pays, visant à promouvoir son image à l'étranger, constitue par exemple une activité à part entière au sein de la presse et du cinéma. À partir des années 1970, l'agence Panorama DDR ${ }^{7}$ se consacre ainsi à l'édition de brochures de propagande sur la RDA à destination de l'étranger et publie des textes dans de nombreuses langues. Le groupe Camera DDR produit quant à lui des documentaires qui poursuivent la même ambition: présenter sous un jour favorable tous les aspects de la vie en RDA dans des moyens métrages traduits dans une dizaine de langues ${ }^{8}$. La qualité matérielle de

5. Lindeperg, p. 192.

6. En proposant une nouvelle traduction, les responsables est-allemands souhaitaient avant tout marquer leur opposition à la RFA en suggérant, à travers le commentaire traduit, que "le ventre de la "bête immonde" [le fascisme] est encore fécond à l'Ouest, définitivement stérile à l'Est ». Ibid., p. 193.

7. Brünner, p. 289-307.

8. Val, p. 338-367. 
ces publications et de ces films témoigne de l'importance des moyens conférés à ces agences et souligne le soin porté à cette communication en direction de l'étranger.

Néanmoins, cette activité de propagande s'accompagne d'un repli sur soi. Malgré les efforts qu'elle déploie, la RDA parvient rarement à accroître sa visibilité et sa reconnaissance à l'Ouest et, ironie de l'Histoire, elle ne retiendra véritablement l'attention de la scène internationale qu'au moment de sa disparition en 1989. Bien que des œuvres culturelles et artistiques de qualité voient le jour en RDA, elles ne touchent qu'un public étranger restreint, constitué soit d'experts dans un domaine, soit de proches du Parti communiste, mais plus rarement le grand public. En outre, lorsqu'elles parviennent à franchir le rideau de fer, leur message est souvent déformé ou mal compris de l'autre côté du Mur. Les films produits en RDA en font régulièrement l'expérience. Que ce soit Sterne (Étoiles, Konrad Wolf, 1959), primé au festival international du film de Cannes, ou le cycle documentaire tourné au Chili par le Studio HES dans les années 1970 et récompensé à plusieurs reprises en France, ces films sont assimilés à des productions respectivement bulgares et chiliennes, mais jamais est-allemandes. Cette non-perception de l'identité est-allemande froisse les responsables de la RDA, qui adoptent alors une position défensive. Après chaque déconvenue, ils optent pour un repli afin d'éviter d'être à nouveau confrontés à cette forme d'indifférence. On assiste à une oscillation constante entre affirmation d'une identité est-allemande singulière et fermeture au monde.

Dans ce contexte complexe, les relations culturelles et artistiques de la RDA avec l'étranger, et en particulier avec l'Europe occidentale, ne peuvent donc être exclusivement le fait des décisionnaires politiques. En revanche, plusieurs personnalités intellectuelles constituent rapidement un réseau de passeurs entre la RDA et les pays occidentaux. Parmi les communistes allemands qui rejoignent la partie orientale de l'Allemagne dans l'immédiat après-guerre, nombreux sont ceux qui, lors de la décennie précédente, avaient fui ce pays, voire l'Europe, pour se réfugier en URSS, aux États-Unis, au Mexique ou ailleurs. Ces trajectoires personnelles leur ont permis de constituer un réseau international sur lequel ils s'appuient à leur retour à Berlin-Est et avec lequel ils continuent d'échanger depuis la RDA. Ces passeurs jovent un rôle crucial dans l'établissement de relations culturelles 
avec l'étranger, tout particulièrement au cours des premières décennies d'existence de la RDA, lorsque celle-ci ne bénéficie encore d'aucune reconnaissance diplomatique officielle sur la scène internationale. À cet égard, le couple formé par Jeanne et Kurt Stern joue un rôle tout particulier. Ces deux communistes convaincus, francophones, traducteurs et scénaristes, exilés à Mexico lors de la Seconde Guerre mondiale, s'insèrent dans le cercle restreint des intellectuels communistes est-allemands et, avec leurs travaux, ambitionnent d'œuvrer au rayonnement de la RDA à l'étranger. En revenant sur leur trajectoire, nous nous interrogerons donc ici sur les contradictions et la complexité de cette ambition.

\section{De l'avant à l'après-guerre: des traducteurs communistes engagés}

Issue d'une famille modeste, Jeanne Machin naît en 1908 dans les Pyrénées. Après avoir été jeune fille au pair en Basse-Saxe, elle poursuit ses études à Berlin durant l'entre-deux-guerres ${ }^{9}$. Elle y fait la connaissance de Kurt Stern (né à Berlin en 1907), également étudiant, qui rejoint le Parti communiste allemand (KPD) en 1927. Par son intermédiaire, Jeanne Machin s'intéresse à son tour à la politique. En 1932, le jeune couple s'installe à Paris, où il se marie. Jeanne Stern rejoint le Parti communiste français (PCF) deux ans plus tard. Elle entreprend la traduction française du roman Der Weg durch den Februar d'Anna Seghers, arrivée en 1935 à Paris avec son mari et ses enfants, alors que l'ascension de Hitler pousse à l'exil de nombreux Allemands; Der Weg durch den Februar est publié par un éditeur français (Les Éditions du Carrefour), chez qui paraissent plusieurs écrivains de l'avant-garde tels que Henri Michaux, Franz Kafka ou Max Ernst. Le Chemin de février sort en 1936, Iorsque la guerre civile éclate en Espagne. Jeanne Stern travaille alors pour l'agence de presse du gouvernement républicain espagnol à Paris, tandis que Kurt Stern rejoint les brigades internationales. À l'issue du conflit et après la défaite des républicains, il est interné dans un camp de prisonniers dans le Sud de la France pendant deux ans. Durant sa détention, il tient un journal en français. 
Le couple parvient à émigrer au Mexique en 1942, tandis que, restés à Berlin, les parents de Kurt Stern, de confession juive, sont déportés et meurent à Auschwitz. À Mexico, Jeanne et Kurt Stern rejoignent Bewegung Freies Deutschland, le mouvement de résistance de l'Allemagne libre, composé d'exilés allemands - communistes et/ou résistants - et particulièrement dynamique en URSS, mais aussi dans d'autres pays comme le Mexique. Ils y retrouvent notamment Anna Seghers, qui préside le cercle Heinrich Heine, dont ils sont membres actifs. Les liens que le couple Stern nove à cette époque avec les autres émigrés et intellectuels allemands seront cruciaux pour la suite de leur parcours.

En 1947, Jeanne Stern entreprend la traduction de Transit, un autre ouvrage d'Anna Seghers. Le roman, plusieurs fois adapté au cinéma depuis lors, raconte le sort des exilés sans papiers réfugiés à Marseille, qui tentent d'échapper à la police française du gouvernement de Vichy pour rallier l'Amérique du Sud. Ce récit est directement inspiré par la propre expérience de Seghers qui, après quelques années à Paris, a rejoint Marseille puis Mexico. Jeanne Stern et Anna Seghers partagent donc une même trajectoire et un engagement politique commun. En outre, l'expérience que relate Transit rencontre un large écho auprès du réseau d'intellectuels communistes exilés dans lequel s'inscrivent les deux femmes. Les liens qu'elles ont tissés en France avant la guerre avec certains éditeurs et artistes ont également contribué à fédérer un cercle de lecteurs admiratifs de l'œuvre de Seghers. La parution française de Transit, à laquelle succède dès 1948 celle de Légendes d'Artémis, répond ainsi à une attente forte de la part d'un certain lectorat.

À cette époque, Anna Seghers et les Stern sont rentrés de Mexico. Jeanne et Kurt Stern passent quelques mois à Paris avant de s'installer à Berlin-Est. Ils rejoignent le parti unique du SED ${ }^{10}$ dès 1946 et emménagent au cœur du lotissement ErichWeinert, l'un des trois quartiers réservés aux intellectuels et artistes socialistes de retour. Pendant que Jeanne Stern traduit Anna Seghers en français, Kurt Stern entreprend la traduction en allemand du Silence de la mer de Vercors. Ce choix d'œuvres littéraires rédigées pendant la guerre, dans la clandestinité ou en

10. Le Parti socialiste unifié d'Allemagne (Sozialistische Einheitspartei Deutschlands, SED) est issu de la fusion entre le Parti communiste allemand (KPD) et le Parti social-démocrate (SPD). 
exil, mettant en scène des personnages confrontés au fascisme, fait directement écho aux préoccupations des contemporains communistes des Stern. Le livre de Vercors, bien que publié clandestinement en 1942, circula d'abord largement parmi les résistants en France, tandis que le récit d'Anna Seghers rappela à tous les intellectuels réfugiés leur propre parcours chaotique. En traduisant ces textes, les Stern prennent une position centrale entre la France et la partie orientale de l'Allemagne. Avec cette activité de "passeurs», ils participent donc de l'engovement général suscité par ces œuvres.

Ces traductions réalisées à la veille de la création de la RDA s'inscrivent dans l'immédiat après-guerre, marqué par la valorisation de la résistance au fascisme pendant la guerre. Les choix de Jeanne et Kurt Stern, guidés par ce contexte, témoignent de leur inscription dans ce réseau d'intellectuels de retour d'exil, profondément marqués par les années de guerre tout en étant pleinement conscients des enjeux pour l'avenir.

\section{De l'adaptation à la réappropriation: les années 1950}

Au début des années 1950, les Stern délaissent leurs travaux de traduction pour se consacrer entièrement à la rédaction de scénarios pour la Deutsche Filmaktiengesellschaft (DEFA), société étatisée de production et de diffusion du cinéma en RDA. Ce passage au cinéma induit dès lors un changement de statut: les Stern ne sont plus de simples passeurs, ils deviennent cette fois les créateurs de contenus originaux. Autant leurs précédentes traductions étaient porteuses d'un esprit d'ouverture, d'une volonté de faire accéder les lecteurs francophones et germanophones à des œuvres provenant d'outre-Rhin, autant les films auxquels ils collaborent sont marqués par l'affirmation d'une identité estallemande forte et par un repli sur les frontières de la RDA.

Ils écrivent d'abord le scénario de Das verurteilte Dorf (Le Village condamné, 1951), premier film réalisé par le cinéaste Martin Hellberg. Le succès de ce long métrage, ouvertement antiaméricaniste" et récompensé à plusieurs reprises, permet au

11. Inspiré d'une histoire vraie, il raconte la révolte des habitants d'un village bavarois que l'armée américaine veut évacuer pour construire un aérodrome militaire. Grâce à la solidarité des communes alentour, les villageois obtiennent finalement gain de cause et découragent l'occupant américain qui abandonne son projet. 
cinéaste d'enchaîner ensuite les tournages. Cet accueil très positif encourage les Stern, qui rédigent alors le scénario de Stärker als die Nacht (Plus fort que la nuit), réalisé par Slatan Dudow ${ }^{12}$ en 1954. Le film s'inscrit pleinement dans l'idéologie officielle qui présente l'antifascisme comme l'identité même de la RDA ${ }^{13}$; son succès en dépasse largement les frontières, et il est positivement accueilli en France, ainsi qu'au festival de Locarno. La confrontation avec la RFA est au cœur du long métrage suivant écrit par Jeanne et Kurt Stern, Das Leben beginnt (La Vie commence, Heiner Carow, 1959). II s'agit cette fois d'une histoire d'amour entre deux adolescents dans le Berlin divisé. Seul le retour de l'héroïne à Berlin-Est offre une issue heureuse à l'intrigue.

Ces trois films de fiction ne laissent aucun doute sur l'engagement total des Stern dans la construction idéologique de l'Allemagne socialiste. Leurs scénarios contribuent en effet pleinement à l'affirmation et à la légitimation du régime estallemand, contre l'Occident en général et contre la RFA en particulier. Cette forme de repli sur l'identité est-allemande et d'opposition frontale à l'Ouest s'inscrit dans le contexte de la guerre froide des années 1950. La RDA apparaît simultanément comme une "vitrine du socialisme», mais elle ne bénéficie d'aucune reconnaissance officielle sur le plan international et se trouve à la frontière directe avec le bloc ovest. La nécessité de s'affirmer comme nation à part entière est donc poussée à son paroxysme. L'évolution de la carrière du couple Stern est elle aussi traversée par cette tension. En effet, leur expérience cinématographique et ce passage à l'écriture de scénarios ne sont pas sans ambiguïtés. Tandis que leurs traductions s'inscrivaient dans un mouvement général d'échange qui ambitionnait précisément de dépasser les frontières (géographique et linguistique), leurs scénarios militent en revanche pour la distinction nette entre une Allemagne antifasciste et une autre qui serait l'héritière du Troisième Reich. De fait, Plus fort que la nuit héroïse les communistes qui ont combattu le fascisme pendant la guerre, tandis que Le Village

12. Slatan Dudow (1903-1963) réalise plusieurs films est-allemands majeurs tels que Unser täglich Brot (Notre pain quotidien, 1949), Familie Benthin (Famille Benthin, 1950) ou encore Der Hauptmann von Köln (Le Capitaine de Cologne, 1956).

13. Le film met en scène le parcours d'un couple hambourgeois engagé dans la résistance communiste dès 1933. Arrêté et exécuté par les nazis peu de temps avant l'arrivée des troupes soviétiques, l'homme envoie à sa femme et à son fils une lettre d'adieux porteuse de l'espoir et de la certitude que son combat et son sacrifice n'auront pas été vains et que l'Allemagne connaîtra bientôt un avenir meilleur. 
condamné et La Vie commence mettent chacun en scène une intrigue s'articulant autour d'une frontière (entre la RDA et l'Ouest dans le premier film, entre Berlin-Est et Berlin-Ouest dans le second), dont la résolution passe par la valorisation du territoire est-allemand.

Cette évolution se révèle pourtant problématique; en effet, en s'emparant pleinement des enjeux qui définissent alors la RDA, les Stern tentent de se réapproprier l'œuvre d'un tiers. Autrement dit, les contenus qu'ils créent pour le cinéma ne sont pas aussi originaux qu'ils prétendent l'être: quelques mois après la sortie du film de Heiner Carow, l'écrivain et scénariste estallemand Jan Petersen, proche de Kurt Stern et d'Anna Seghers, accuse le couple Stern d'avoir plagié deux de ses œuvres ${ }^{14}$. Ses arguments sont pour le moins probants, si bien que le Deutscher Schriftstellerverband (association des écrivains allemands) se réunit le 10 janvier 1961 pour trancher. Toutes les accusations de plagiat sont finalement rejetées, mais a priori pour faire bonne figure et ne pas jeter le discrédit sur des scénaristes récompensés par plusieurs prix prestigieux en RDA. Les preuves avancées par Jan Petersen laissent pourtant peu de place au doute et l'attitude des Stern amène à les croire coupables de plagiat: ils récidivent presque immédiatement, en s'attribuant à nouveau l'œuvre d'autrui dès le début des années 1960. Ils travaillent avec tant d'ardeur à l'adaptation allemande d'un documentaire réalisé par Joris Ivens au moment de la guerre d'Espagne, qu'ils effaceront momentanément son nom du générique ${ }^{15}$.

\section{Porter la parole communiste et se taire}

Après ce passage par le cinéma, le couple revient à la traduction dans les années 1960. Jeanne Stern s'attelle à proposer une version française de classiques de la littérature est-allemande, notamment Bertolt Brecht, Friedrich Engels, Alexander Abusch et de nouveau Anna Seghers. Si ces œuvres font écho à l'engagement politique des Stern, ce retour à cette activité de passeurs entre l'Est et l'Ouest et les collaborations qu'il implique avec leurs

14. Lettre de Jan Petersen à Alexander Abusch, juillet 1960.

15. «Nach der Zulassung fiel auf, dass der Titel sich nur auf Kurt und Jeanne Stern bezog und Joris Ivens außen vor blieb, obwohl die knappe Hälfte des Films aus der gekürzten Synchronfassung von Ivens' Spanische Erde bestand ». Jordan, p. 246. 
homologues étrangers s'inscrivent cette fois dans une volonté de porter de grandes voix d'intellectuels communistes à l'extérieur de la RDA. Jeanne Stern travaille ainsi avec Gilbert Badia et Geneviève Serreau sur certaines pièces de théâtre de Brecht. Badia est l'un des membres des Échanges franco-allemands, une association créée par des germanistes français dans le but de militer en faveur de la reconnaissance diplomatique de la RDA par la France. De son côté, Kurt Stern sert d'intermédiaire à des projets de collaborations cinématographiques entre la France et la RDA et accepte d'apparaître dans le premier (et l'un des très rares) documentaire français consacré à la RDA ${ }^{16}$.

Leurs choix coïncident donc parfaitement avec la ligne officielle du SED. Les Stern ne remettent d'ailleurs à aucun moment en question les décisions politiques du Parti. Cette adhésion leur permet de gagner suffisamment la confiance des fonctionnaires est-allemands pour être autorisés à voyager à l'étranger; ils se rendent ainsi au Vietnam en 1968 et en rapportent un recueil de témoignages sur la guerre ${ }^{17}$. Par ailleurs, leur œuvre commune en tant que scénaristes, traducteurs et écrivains est couronnée en 1970 par le prix Heinrich Mann, attribué par l'Akademie der Künste (Académie des arts) de Berlin ${ }^{18}$. Plus que leur carrière en tant que traducteurs et scénaristes, c'est peut-être leur fidélité à la RDA, leur absence de prise de parole à l'encontre de la politique menée par le SED et leur silence qui contribuent véritablement à les intégrer au cercle restreint des compagnons de route de la RDA. Comme le décrit Sonia Combe au sujet de Jürgen Kuczynski, une autre figure intellectuelle communiste est-allemande, ces personnalités "refusai[ent] d'admettre l'évidence: que le Parti ne pouvait se réformer de l'intérieur». Les prix décernés aux Stern, les liens privilégiés qu'ils étaient autorisés à entretenir avec l'étranger, et en particulier avec la France, étaient la contrepartie de leur adhésion totale à la RDA et de leur silence.

Kurt Stern meurt en septembre 1989, quelques semaines avant la chute du Mur, et Jeanne Stern, neuf ans plus tard. En 2006 ,

16. Derrière le Mur, Mario Marret, Jacqueline Meppiel, 1967.

17. Kurt Stern, Reisfelder-Schlachtfelder. Augenzeugenbericht über Vietnam im Krieg, Halle (Allemagne), Mitteldeutscher-Verlag, 1968. Ensemble, ils rédigent également Bevor der Morgen graut: Vietnam zwischen Krieg und Sieg, Berlin, Neves Leben, 1969.

18. Les archives du couple y sont d'ailleurs conservées: https://archiv.adk.de/bigobjekt/12249, consulté le 13 mai 2019. 
leur fille, Lucienne Steinitz, traduit en allemand le journal rédigé par Kurt Stern lors de son internement en France en 1939-1940. La préface de l'ouvrage, signée par Christa Wolf, rappelle l'importance des Stern dans le cercle intellectuel communiste de la RDA, bien que leurs noms soient aujourd'hui tombés dans l'oubli.

\section{perrineval@wanadoo.fr}

Perrine Val est l'auteure d'une thèse de doctorat en histoire du cinéma intitulée "Les relations cinématographiques entre la France et la RDA : entre camaraderie et exotisme (1946-1992)» et soutenue en mai 2018 à I'Université Paris 1 Panthéon Sorbonne. Ses recherches montrent comment le cinéma constitua un espace de rencontre entre l'Est et l'Ouest pendant la guerre froide. Elle a notamment publié «Diffusion et réception du cinéma est-allemand en France» (in Allemagne d'aujourd'hui $n^{\circ} 198$ ) et «Von Ost nach West: Filme aus der DDR in Frankreich» (avec Matthias Steinle, in Skyler Arndt-Briggs et al., DEFA International. Grenzüberschreitende Filmbeziehungen vor und nach dem Mauerbau)

\section{SOURCES CITÉES}

BARJONET Aurélie, Zola d'Ouest en Est. Le naturalisme en France et dans les deux Allemagnes, Rennes (France), PUR, 2010.

BUFFET Cyril, Défunte DEFA: histoire de l'autre cinéma allemand, Paris, Cerf, coll. « $7^{\circledR}$ Art», 2007.

BRÜNNER Thomas, «Die Presseagentur "Panorama DDR". Anspruch und Praxis der "Auslandsinformation" in Frankreich 1963-1973", in KWASCHIK Anne et PFEIL Ulrich (dir.), Die DDR in den deutsch-französischen Beziehungen, Bruxelles, Peter Lang, 2013, p. 289-307.

COMBE Sonia, «Le silence comme éthique? Jürgen Kuczynski (1904-1997) : tentative de portrait», Cahiers d'histoire. Revue d'histoire critique, 120, 2013, p. 137-154.

JARAUSCH Konrad H., «Die DDR-Geschichtswissenschaft als "Meta-Erzählung”, in SABROW Martin (dir.), Verwaltete Vergangenheit. Geschichtskultur und Herrschaftslegitimation in der DDR, Leipzig (Allemagne), Akademische Verlagsanstalt, 1997, p. 19-34.

JORDAN Günter, Unbekannter Ivens. Triumph, Verdammnis, Auferstehung. Joris Ivens bei der DEFA und in der DDR 1948-1989, Berlin, DEFA-Stiftung, 2018.

LINDEPERG Sylvie, «Nuit et Brouillard». Un film dans l'histoire, Paris, Odile Jacob, 2007.

POPA Ioana, Traduire sous contraintes. Littérature et communisme (1947-1989), Paris, CNRS Éditions, 2010.

SCHÄTTE Ulrike, «Ich bin immer mit meinen Diplomen gereist. Jeanne Stern: ein Leben zwischen Frankreich, Deutschland und Mexico», in ila, 212, p. 48-52, 1998, www.ila-web.de/ ausgaben/212/ich-bin-immer-mit-meinen-diplomen-gereist, consulté le 13 mai 2019. 
SCHENK Ralf (dir.), Das zweite Leben der Filmstadt Babelsberg. DEFA-Spielfilme 1946-1992, Berlin, Henschel, 1994.

STEINLE Matthias, Vom Feindbild zum Fremdbild. Die gegenseitige Darstellung von BRD und DDR im Dokumentarfilm, Konstanz (Allemagne), UVK, coll. «Close up», 2003.

STERN Kurt, Was wird mit uns geschehen? Tagebücher der Internierung 1939 und 1940, Berlin, Aufbau-Verlag, 2006.

VAL Perrine, Les relations cinématographiques entre la France et la RDA: entre camaraderie et exotisme (1946-1992), thèse de doctorat soutenue à l'université Paris 1 Panthéon Sorbonne, 2018. WENKEL Christian, Aufder Suche nach einem "anderen Deutschland". Das Verhältnis Frankreichs zur DDR im Spannungsfeld von Perzeption und Diplomatie, Munich (Allemagne), De Gruyter/ Oldenburg, 2014. 